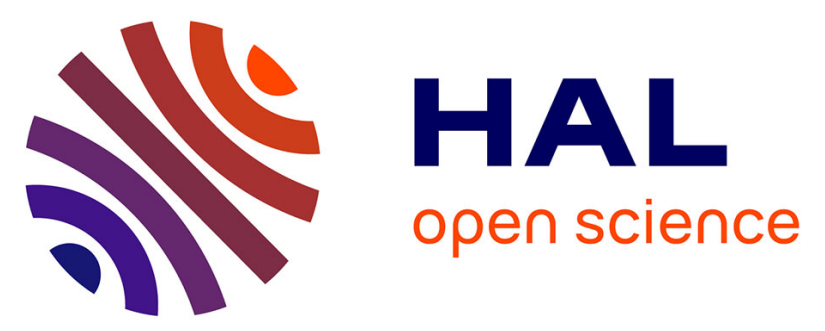

\title{
Perspectives for integrating human and environmental risk assessment and synergies with socio-economic analysis
}

Alexandre R.R. Pery, Gerrit Schuurmann, Philippe Ciffroy, Michael Faust, T. Backhaus, Lothar Aicher, Enrico Mombelli, Cléo Tebby, Mark Cronin, Sylvie

Tissot, et al.

\section{To cite this version:}

Alexandre R.R. Pery, Gerrit Schuurmann, Philippe Ciffroy, Michael Faust, T. Backhaus, et al.. Perspectives for integrating human and environmental risk assessment and synergies with socio-economic analysis. Science of the Total Environment, 2013, 456, pp.307-316. 10.1016/j.scitotenv.2013.03.099 . ineris-00961805

\section{HAL Id: ineris-00961805}

https://hal-ineris.archives-ouvertes.fr/ineris-00961805

Submitted on 20 Mar 2014

HAL is a multi-disciplinary open access archive for the deposit and dissemination of scientific research documents, whether they are published or not. The documents may come from teaching and research institutions in France or abroad, or from public or private research centers.
L'archive ouverte pluridisciplinaire HAL, est destinée au dépôt et à la diffusion de documents scientifiques de niveau recherche, publiés ou non, émanant des établissements d'enseignement et de recherche français ou étrangers, des laboratoires publics ou privés. 
Perspectives for integrating human and environmental risk assessment and synergies with socio-economic analysis

A.R.R. Péry ${ }^{\mathrm{a},}$, G. Schüürmann ${ }^{\mathrm{b}, \mathrm{c}}$, P. Ciffroy ${ }^{\mathrm{d}}$, M. Faust ${ }^{\mathrm{e}}$, T. Backhaus ${ }^{\mathrm{e}}$, L. Aicher ${ }^{\mathrm{f}}$, E.

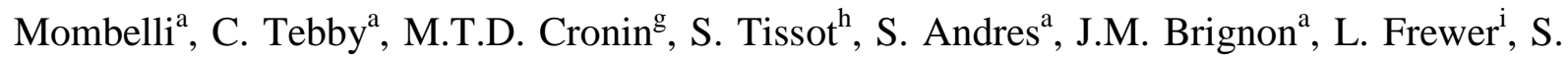

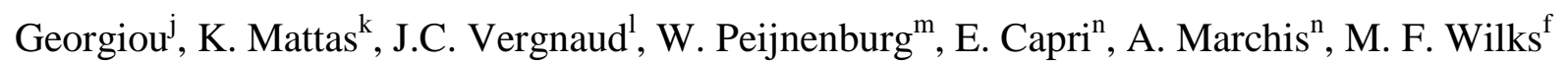
${ }^{a}$ INERIS, Parc Alata, BP2, 60550 Verneuil-en-Halatte, France

${ }^{\mathrm{b}}$ UFZ Department of Ecological Chemistry, Helmholtz Centre for Environmental Research, Permoserstr. 15, 04318 Leipzig, Germany

${ }^{\mathrm{c}}$ Institute for Organic Chemistry, Technical University Bergakademie Freiberg, Leipziger Str. 29, 09596 Freiberg, Germany

${ }^{\mathrm{d}}$ EDF, Division Recherche et Développement, Chatou, France

${ }^{\mathrm{e}}$ Faust \& Backhaus Environmental Consulting GbR, Fahrenheitstrasse 1, 28359 Bremen, Germany

${ }^{\mathrm{f}}$ Swiss Centre for Applied Human Toxicology, University of Basel, Switzerland

g School of Pharmacy and Chemistry, Liverpool John Moores University, Byrom Street, Liverpool, L3 3AF, England

${ }^{\mathrm{h}}$ SYNGENTA Crop protection AG, Schwarzwaldallee 215, CH-4058 BASEL, Switzerland

${ }^{\mathrm{i}}$ Food and Society Centre for Rural Economy, School of Agriculture, Food and Rural Development, Newcastle University, Agriculture Building, Newcastle upon Tyne NE1 7RU, United Kingdom

${ }^{\mathrm{j}}$ Chemicals Regulation Directorate, Health and Safety Executive, G/NW Rose Court 2 Southwark Bridge, London, SE1 9HS, United Kingdom

k Aristotle Univeristy of Thessaloniki, School of Agriculture, Dept. of Agricultural Economics, P.O. Box 225, 541 24, Thessaloniki, Greece 
${ }^{1}$ Centre d'Economie de la Sorbonne, Université Paris 1 - Sorbonne, France

${ }^{\mathrm{m}}$ RIVM - National Institute for Public Health and the Environment, Laboratory for Ecological Risk Assessment, Bilthoven, the Netherlands

${ }^{\mathrm{n}}$ OPERA Research Centre, Universita Cattolica del Sacro Cuore. Via E. Parmense 84. 29100 Piacenza. Italy

${ }^{*}$ Corresponding author at: INERIS, Parc Alata, BP2, 60550 Verneuil-en-Halatte, France. Tel.: +33445561 26; fax: +3344556800.

E-mail address: alexandre.pery@ineris.fr (A. Péry). 


\section{ABSTRACT}

For more than a decade, the integration of human and environmental risk assessment (RA) has become an attractive vision. At the same time, existing European regulations of chemical substances such as REACH (EC Regulation No. 1907/2006), the Plant Protection Products Regulation (EC regulation 1107/2009) and Biocide Regulation (EC Regulation 528/2012) continue to ask for sector-specific RAs, each of which have their individual information requirements regarding exposure and hazard data, and also use different methodologies for the ultimate risk quantification. In response to this difference between the vision for integration and the current scientific and regulatory practice, the present paper outlines five medium-term opportunities for integrating human and environmental RA, followed by detailed discussions of the associated major components and their state of the art. Current hazard assessment approaches are analyzed in terms of data availability and quality, and covering non-test tools, the integrated testing strategy (ITS) approach, the adverse outcome pathway (AOP) concept, methods for assessing uncertainty, and the issue of explicitly treating mixture toxicity. With respect to exposure, opportunities for integrating exposure assessment are discussed, taking into account the uncertainty, standardization and validation of exposure modelling as well as the availability of exposure data. A further focus is on ways to complement RA by a socio-economic assessment (SEA) in order to better inform about risk management options. In this way, the present analysis, developed as part of the EU FP7 project HEROIC, may contribute to paving the way for integrating, where useful and possible, human and environmental RA in a manner suitable for its coupling with SEA.

Keywords:

Human risk assessment

Environmental risk assessment

Socio-economic analysis

Hazard assessment

Chemical exposure

Integration 


\section{Introduction.}

Current risk assessment (RA) policies, processes and practices have been established through extensive cumulative experience of scientists from many disciplines and in different countries over decades, resulting in national and international regulations for a wide range of stressors. Nevertheless, RA is facing increasing challenges, in particular with regard to increasing scientific complexity, public perception and resourcing.

On the one hand, the categories and number of substances for which a human and/or environmental RA is required will continue to increase substantially due to revised legislation (e.g. REACH: Registration, Evaluation, Authorisation and Restriction of Chemical substances ; more complete ecological risk assessment is expected for pharmaceuticals in the next few years). The need to assess the impacts of multiple stressors and the toxicity of mixtures adds additional complexity. On the other hand, non-scientific considerations such as budget restrictions or political and public pressure to reduce the number of animal tests for the purpose of safety assessments also drive further developments in RA.

Taken together, these developments create the need for better exploiting all currently existing data, and it is assumed that a more integrated approach to RA is part of the solution. In theory, the potential benefits of Integrated Risk Assessment (IRA) have been recognized for more than a decade. Several projects have been carried out including a joint WHOIPCS/US-EPA project (WHO, 2001) and a range of EU projects (for instance INTARESE (http://www.intarese.org/), HEIMTSA (http://www.heimtsa.eu/), 2-FUN (http://www.2fun.org/), NOMIRACLE (nomiracle.jrc.ec.europa.eu/)) have already addressed integrated risk assessment. However, the impact of those initiatives on the development of IRA-approaches has been limited so far, and today no explicit legal mandates for IRA exist. Therefore, there is not yet consensus among experts about the scale of implementation achieved so far. This is 
partly because there is no harmonized definition and understanding of what IRA means in practice.

As a step towards a common understanding of integrated risk assessment, and to assess how risk analysis and socio-economic analysis can benefit from each other, this paper reviews perspectives in integrated hazard and exposure assessment. The work presented here is derived from reviews performed by partners of the HEROIC project (Health and Environmental Risks: Organisation, Integration and Cross-fertilisation of Scientific Knowledge, FP7, Grant Agreement no. 282896), from discussions during the first expert meeting within the HEROIC project, organized at INERIS (Verneuil-en-Halatte, FRANCE) the $12^{\text {th }}$ and $13^{\text {th }}$ of April 2012, and from a meeting with the Scientific Advisory Board of HEROIC on 21 September 2012 in Basel, Switzerland. The HEROIC project was established to promote strengthening of the interfaces between human and environmental endpoints within RA procedures, thus paving the way towards an integrated approach where the exploitation of mechanistic information across human and environmental toxicology becomes a focal theme. Below, the HEROIC view of the opportunities for integrated risk assessment is outlined, followed by more detailed discussions of current developments in the areas of exposure, hazard and risk assessment. Particular emphasis is placed on integrated testing strategies with explicit consideration of mixture toxicity and approaches to augment RA by a socio-economic assessment.

With the implementation of REACH on June 1, 2007, the concept of integrated testing strategies (Ahlers et al., 2008; Bradbury et al., 2004; Combes and Balls, 2005; OSIRIS, 2011) became an official part of the European-wide regulation of industrial chemicals. As outlined in more detail below, the ITS approach builds on the combined information content extractable from non-test and test data including information from non-standard (non-guideline) 
sources, confining the additional generation of animal-based data to the minimum required for completing the assessment of interest.

While the ITS concept mainly focuses on integrating different types of information for assessing the human or environmental hazard potential of chemical substances, an extension of this approach to exposure assessment and to eventually assessing human and environmental risk is the subject of the integrated RA framework. Within HEROIC, the following five medium-term opportunities for RA integration have been identified:

- Employ the mode of action (MOA), or eventually the adverse outcome pathway (see below) as a trigger and criterion for the mutual exploitation of environmental and human toxicology data.

- Explore the applicability of existing weight-of-evidence (WoE) schemes for integrating environmental toxicity data into human health assessments and vice versa, considering appropriate means for assessing the level of confidence (or level of uncertainty) of the individual data as well as of their combined content.

- Provide a framework for merging risks associated with individual exposure routes into an overall risk reflecting the combined exposure.

- Include an explicit consideration of mixture toxicity effects into human and environmental risk assessment

- Augment the risk assessment process by a socio-economic analysis targeted to inform about the pros and cons of possible risk management options.

At present, these five issues are considered as a roadmap of opportunities toward building integrated RA procedures, to which HEROIC will contribute through scientific networking activities on a European scale and across academia, business and regulatory authorities. 


\section{Perspectives on integrated hazard assessment contributing to integrated risk} assessment

Integrated hazard assessment relates to the possibility of combining information content from non-test and test data including information from non-standard (non-guideline) sources or from species different from the targeted one (in particular when integrating environmental toxicity data into human health assessments and vice versa). The success of such integrated hazard assessment relies on four conditions. First the amount of available data should be sufficient, which means that availability of hazard data should be enhanced. Second, a framework is needed which must be able to integrate different information for a given endpoint. This is what is expected from the Adverse Outcome Pathways (AOPs, Ankley et al., 2010). Third, hazard assessors should have appropriate tools, in particular with regard to modeling, for the integration and the extrapolation. Fourth, managing different sources of data (measured, extrapolated, non-test data) requires the development of methods to assess the confidence level of data, which would contribute to building relevant weight-of-evidence (WoE) approaches.

\subsection{Improving data availability for risk assessors}

The benefit of sharing toxicity and exposure data and making them available is largely recognized. However, most of the data used in risk assessment for substances and products remain confidential, in particular due to intellectual property issues.

There are many reasons for the lack of publicly available data. They may only be available as paper copies not suitable for immediate electronic storage or sharing. Moreover, there could be no suitable platform to share data (cf. inventory with separate databases), or there is little or no compatibility of databases, data structure or descriptors. Significant efforts 
are now being undertaken to at least partially answer the need to share information and develop common templates and descriptors. Examples include TOXNET (http://toxnet.nlm.nih.gov/), e-Chem portal (www.echemportal.org/), and IUCLID (iuclid.eu/). The OECD QSAR Toolbox also proposes a way to link databases together. The limitation is that so far only a part of the information is accessible via these formats, and only information developed within a regulatory context is included. Pharmaceuticals, plant protection products (PPP) and biocides are not included in shareable formats.

A practical solution to overcome the issue of data confidentiality has been proposed in the EU IMI project "Integrating bioinformatics and chemoinformatics approaches for the development of expert systems allowing the in silico prediction of toxicities" (eTOX) which includes academic institutions, small and medium-sized enterprises (SMEs), and large pharmaceutical companies (Briggs et al., 2012). The companies participating in the project consortium share confidential data to build predictive tools or perform compound comparisons, but the access is restricted to one of the SMEs, called "honest broker". Data confidentiality is not only limited to private companies and registration. Distributed StructureSearchable Toxicity (DSSTox) Database Network (www.epa.gov/ncct/dsstox/) is a project of EPA's National Center for Computational Toxicology, which aims to build a public toxicity database for improved structure-activity and predictive toxicology capabilities. Among the reasons why a potential author may consider publishing a database on DSSTox are increased recognition for authorship and expertise relative to a toxicity database; increased visibility and use of the database by a wide range of users and scientific disciplines; ability to merge the database with many other toxicity databases in structure-searchable format; potential for collaborations and user feedback. In order to foster data sharing further, it could be useful to create a peer-review journal dedicated to publishing data that can be shared. Any use of data published in this journal would require the citation of the associated article. 


\section{2. $\quad$ Non-test tools to extrapolate in hazard assessment}

Non-test methods can permit the extrapolation of available toxicological information, between chemicals, between species and between different levels of biological organization, and support integrated risk assessment by generating additional information.

QSAR models and read-across can be used in order to extrapolate data from one chemical to another. Read-across is based on experimental data and expert judgment, which is different from QSAR models, which propose a regression-based relationship between the values of relevant descriptors and the toxicological response. Read-across requires the assessment of chemical similarity. The latter can be defined in a number of ways, and in fact can be understood as covering different components (e.g. structural similarity, presence of certain structural alerts, physicochemical profile), the selection of which depends on the target property of interest. Typically, some method of assessing structural similarity (e.g. finding structural analogs or employing atom-centered fragments (Kühne et al., 2009)) forms the starting point. Within the toxicological context further issues such as bioavailability (characterized through a certain physicochemical profile (Dimitrov et al., 2005)), presumed mechanism and mode of action, potential involvement of metabolic conversion leading to toxification, detoxification or more efficient elimination can be invoked. An analysis of the REACH dossiers submitted to ECHA during the first registration period between 1 June 2008 and 28 February 2011 (high production volume chemicals and substances of very high concern) revealed that among the non-test methods, read-across has by far had largest use, while QSAR-based information apparently played only a minor role (Spielmann et al., 2011). At the same time, current practice indicates that there is a lack of experience in regulatory contexts with read-across despite the fact that the general principles of this predictive 
approach are already covered by existing guidelines (OECD, 2007; OECD, 2009). Within these documents it is acknowledged that by means of a learning-by-doing approach, the experience gained in application of read-across will lead to further improvements and changes of existing guidance documents. Indeed, the majority of the examples that the available documents provide are mainly focused on toxicological endpoints that are governed by rather well understood mechanisms for which there are well populated databases (e.g. skin sensitization, Ames genotoxicity, aquatic toxicity). In this respect the ECETOC technical report on read-across (ECETOC, 2012) provides a new analysis on the impact of predictions based on the analogue approach by highlighting two facts: data on toxicokinetics can be a key piece of evidence when substantiating a read-across approach and these data can be collected during standard toxicological studies with very limited consequences on animal welfare. Moreover, this report also pointed out that, at the current time, read-across is limited to small chemical categories in order to counterbalance lack of mechanistic knowledge about certain endpoints. According to the ECETOC report this limitation will be removed in the next future thanks to programs such as Toxicity testing in the $21^{\text {st }}$ century (Bhattacharya et al., 2011).

Integration of data obtained for species or biological levels of organization different from the target requires the assessment of the dose response for both toxicokinetics (exposure concentration at local or systemic level for a given dose) and toxicodynamics (local or systemic dose-response depending on the endpoint considered). Comparative toxicokinetics is based on physiologically-based toxicokinetics (PBTK) models. A PBTK model consists of a series of mathematical equations which, based on the specific physiology of an organism and on the biophysical properties of a substance, are able to describe the absorption, distribution, metabolism and elimination (ADME) of the compound within this organism. Inter-species extrapolation is by far the most common and the most documented PBTK usage in pharmacokinetics (Nestorov, 2003). The scaled species-specific information is most often 
related to physiological parameters, even if some compound specific information, obtained in vitro for instance, can be used. In terms of toxicodynamics, a way forward could be to promote using mechanistic information available for one sector (e.g. endocrine disruption in aquatic toxicology) to another sector (e.g. human health), and thus more generally to exploit toxicological information across traditional boundaries. AOP approaches should help in achieving the goal. Indeed, interspecies extrapolation would be facilitated through the identification of common key receptors or the use of comparative genomics that would indicate the plausibility of common key events (OECD, 2011).

There are only a few examples for which PBTK models were used to extrapolate in vitro response to in vivo hazard assessment (e.g. Punt et al., 2011). In general, the predictions over-estimated the toxicity, which could be both due to overestimation of the effects in the in vitro systems and uncertainty on the toxicokinetics parameters. Thus, there are improvements to be made both in the experimental design and in the accurate calibration of toxicokinetics models. As stated by Punt et al. (2011), more examples that provide proof of principle for deriving in vivo dose-response curves based on in vitro assays and PBK modeling techniques will be necessary for integrated hazard assessment.

\subsection{Integrated Testing Strategies}

The introduction of the concept of Integrated Testing Strategies (ITS) in the preparation phase of REACH constituted a paradigm shift regarding the use of alternative methods for the hazard assessment of chemical substances: The original 3R idea of one-to-one replacements of animal tests by in vitro tools was converted into the broader concept of replacing one animal test by the combined use of several non-animal methods. Respective ITS methods and 
guidance have been developed in the EU-funded project OSIRIS for both human and environmental endpoints (OSIRIS, 2011).

In an ITS, both non-test and test data may be included. In addition to methods providing data for the physicochemical properties and the human or environmental toxicity endpoint of interest, information theory tools such as (qualitative or quantitative) weight of evidence and consensus modelling can be employed. This will help to unravel the combined information content, and to possibly augment the resulting evaluation with a certain level of probability. Typical ITS components for providing non-test and test data can be grouped into three classes, with in vivo methods being understood as being last resort:

Non-test (in silico) methods

- $\quad$ Analysis of existing test data

- $\quad$ Read-across

- Chemical categories

- $\quad$ Structural alerts

- $\quad$ QSAR (quantitative and qualitative structure-activity relationships)

- $\quad$ Exposure modelling

- $\quad$ TTC (thresholds of toxicological concern)

Non-animal tests

- In chemico (chemoassays)

- In vitro (bioassays)

- Omics (transcriptomics, proteomics, metabolomics)

- $\quad$ Exposure monitoring

In vivo methods

- $\quad$ Lower-tier tests (short-term, single organisms)

- $\quad$ Higher-tier tests (long-term, population, community) 
Regarding the prediction of physicochemical properties from chemical structure, the reader is referred to an earlier review (Schüürmann et al., 2007), keeping in mind that some of these properties also trigger the need for animal testing (e.g. bioconcentration testing becomes relevant if the logarithmic octanol/water partition coefficient, $\log K_{\mathrm{ow}}$, is above 3 , because this indicates that the substance may bioaccumulate to a significant degree (ECHA, 2012)). Note, however, that seemingly minor issues such as wrong tautomers - a problem sometimes overlooked in existing chemical inventories - may result in severe prediction errors (Thalheim et al., 2010), which is also a concern when exceeding the QSAR application domain (Dimitrov et al., 2005; Kühne et al., 2009).

One of the main challenge in ITS is to provide information when experimental data are missing. Examples of in silico ITS components for human and environmental toxicology include structural alerts for the predictive identification of high-concern compounds (Benigni and Bossa 2011; Mekenyan et al., 2010; von der Ohe et al., 2005), and a first fully computerized read-across approach for predicting the acute fish toxicity of organic compounds (Schüürmann et al., 2011). Within more narrow mechanistic domains such as Michael-acceptor electrophiles, $\alpha$-alkyl nitrosamine pro-electrophiles and primary aromatic amines, in silico tools employing computational chemistry may inform about reactivity-driven toxicity (Mulliner et al., 2011, Wondrousch et al., 2010) as well as about predominant metabolic activation pathways (Ji and Schüürmann, 2012, 2013).

Reactive toxicity can also be sensed experimentally through chemoassays, focusing on covalent binding either to proteins or to the DNA (Böhme et al., 2010, Lalko et al., 2011, Schwöbel et al., 2011, Thaens et al., 2012). In this context, a further non-animal approach is given by aquatic bioassays and their potential for identifying reactive-toxic compounds through their excess toxicity as opposed to baseline narcosis (Blaschke et al. 2012, Schramm 
et al., 2011), thus supporting the identification of structural alerts as the basis for the development and extension of respective in silico models.

For a given endpoint and substance of interest, the ITS procedure starts by collecting all potentially relevant data. Besides data generated according to the respective guidelines (e.g. OECD guidelines), also non-standard data may be taken into account, keeping in mind that in the case of toxicological or ecotoxicological endpoints, additional in vivo testing should be considered only as a last resort. Subsequently, the collected data need to be assessed with regard to their reliability and relevance for the intended purpose, which in our context would be risk assessment, but could also be classification and labelling. The final step is a weight-ofevidence (WoE) evaluation (Weed, 2005) of the combined information content, taking into account methods to assess the overall level of uncertainty or level of confidence, and considering opportunities for consensus modelling. Respective computerized ITS schemes for the human endpoints skin sensitization, mutagenicity and carcinogenicity (Buist et al., 2013), repeated-dose toxicity (Tluczkiewicz et al., 2013), and for the environmental endpoints aquatic toxicity and bioconcentration are available through the free-of-charge OSIRIS webtool (OSIRIS, 2011).

Regarding the RA context, the ITS approach focuses on integrating the hazard assessment procedure, while the still broader scope of an integrated RA is understood to include methods for addressing cumulative exposure and mixture toxicity (see below) and possibly further levels of integration.

\subsection{Adverse Outcome Pathways (AOPs)}

In response to apparent differences in understanding of the terms mode of action (MOA) and mechanism of action, Ankley et al. (2010) introduced the term adverse outcome 
pathway (AOP) as a unifying concept. While the term MOA was originally introduced as a sequence of key events at the biochemical or physiological level leading to the toxicological response, the mechanism of action can be understood as a comprehensive description of all relevant steps from the molecular initiating event to the toxicity at the phenomenological level (Ankley et al., 2010; Carmiachael et al., 2011; ECETOC, 2006, 2007).

In practice, however, a MOA is often confined to a selected response at the biochemical or physiological level (e.g. oxidative uncoupling) or simply refers to the molecular initiating event (e.g. DNA-reactive mode of mutagenicity), whilst a mechanism of action typically refers to a selected series of molecular-level steps before the final outcome (e.g. dioxin toxicity: association with the Ah receptor in the cytosol, translocation to the nucleus, association with the Ah receptor nuclear translocator (ARNT), binding at DNA inducing expression of mRNA and protein synthesis).

In this context, the AOP was defined as a conceptual construct that represents the sequence of events from the molecular initiating event to the adverse outcome at the level of interest (Ankley et al., 2010). AOPs span multiple levels of biological organization (OECD, 2012). In Figure 1, the AOP approach is illustrated by a sequence of major steps involved in the development of a toxicological response.

\subsection{Accounting for uncertainty in integrated hazard assessment}

Integrative methods such as those presented previously allow the use of all possible sources of information and data by using measured or extrapolated data, or data generated through modeling approaches. As the nature of these data is very diverse, the integration or weightings of the different information should account for the level of confidence or certainty of the data relative to the ultimate prediction of an adverse effect. If predictive uncertainty for 
point estimates were derived in the form of probabilistic distributions for in vivo tests, in vitro tests and non-test methods, the relevance of ITS to support decisions would be improved (Jaworska et al., 2010, Péry et al., 2010).

Statistical methods are available or under development to assess the uncertainty of toxicological and ecotoxicological data, QSAR predictions and reference standard tests. Indeed, it is important to note that even data from reference tests have an inherent uncertainty (Péry et al., 2010). For instance, Janer et al. (2007) showed that the difference between two NOAELs for the same subchronic study performed for the same chemical could be up to a factor around 10. The calculation of a confidence interval for a test method is almost straightforward now. For instance, the confidence in BMDx (benchmark dose corresponding to $\mathrm{x} \%$ of effect) can be calculated through existing software (Davis et al., 2011). In particular, the estimation of a BMD 95\% lower bound confidence limit (BMDL) permits accounting for study quality (i.e. sample size).

The uncertainty in predictions of non-test methods such as QSAR models should be verified, ideally with an external test set (i.e. a set of chemicals that was not used in the model development), provided that the latter covers the relevant chemical domain properly. In case a sufficient number of new data for compounds with a sufficient variation in chemical structure are not available, cross-validation, bootstrapping or re-sampling offer useful alternatives. In any case, the uncertainty associated with a QSAR prediction cannot be expected to be lower than the uncertainty of the data on which the QSAR model is based. Recent developments in QSAR models now permit better estimates of the uncertainty in the prediction. They consider the model itself as well as the distance between the query molecule and the applicability domain for which the model is valid (Pery et al., 2009; Tebby and Mombelli, 2012). Since the applicability domain is fuzzy to some degree, a methodology based on atom-centered fragments (ACFs) has been developed to identify intermediate levels of belonging (Kühne et 
al. 2009). Application of this approach to existing QSAR models that were confronted with data published after their development demonstrated an external performance similar to the original calibration performance only for the ACF-based category "inside", a moderate but significant increase in the average prediction error for compounds "borderline inside", and further stepwise decreases in the prediction performance for compounds of the "borderline outside" and "outside" categories. It must also be noted that even inside the applicability domain, predictive uncertainty can vary especially in case of chemicals that are not uniformly distributed in the chemical space defined by the selected physicochemical descriptors (Schultz et al., 2007).

\section{Perspectives in integrated exposure assessment}

\subsection{What is integrated exposure assessment?}

By definition, exposure assessment establishes the link from (intentional or unintentional) emissions of chemicals into the environment to exposure of biological targets. These latter can be considered at different scales: ecosystems, communities, populations, whole organisms, organs, tissues, cells. Exposure assessment is, by essence, integrated, because it has to account for all the interacting compartments and physico-chemical phenomena involved in the fate of the chemical under investigation. In the Emission-to-target chain several steps can be defined (Figure 2). First, environmental transport and fate describe or predict the chemical concentration in physical media such as surface water, groundwater, (indoor and outdoor) air, soils, sediments or surfaces. In the specific case of persistent pollutants that can move over long distances, long-range transport models are needed to evaluate the transfer of chemicals to remote regions. Fate models are essentially based on 
biodegradation and partitioning properties of chemicals that govern their distribution among physical media. Second, bioaccumulation, and eventually bioconcentration are used to estimate the internal concentration in biological media that form part of the food chain. Third, for human exposure assessment, scenarios are constructed to estimate the potential exposure via ingestion, inhalation and dermal contact for chemicals present in the workplace or in the environment. This evaluation involves assumptions related to human behaviour, in particular the diet. Finally, once in the body, the chemical is subject to pharmaco-kinetic processes that govern its distribution in organs and tissues, its metabolism and elimination. The integration of all steps cannot be achieved without the support of mathematical models, able to deal with different space and time scales.

With an ultimate goal of linking hazard and exposure assessment, the last step relative to the prediction of concentration in the body, in particular at the level of target organs or tissues, is crucial. At this step, all routes of exposure should be considered simultaneously, because the sum of internal doses from the different routes may not actually account for this multi-route exposure because of non-linear phenomena (saturation of liver metabolism, for instance). With regard to the internal concentration prediction, threshold levels at organ or tissue level (i.e. tolerable concentrations in target organs and tissues) should be available. At the moment, they are available only for few chemicals. However, with the progress in mechanistic toxicology, as well as the development of biomonitoring programmes, it can be expected that 'equivalent biomonitoring reference Doses' will be defined in the future. Some approaches based on reverse pharmacokinetic modeling are thus developed to calculate chemical- and tissue-specific concentration thresholds (or 'biomonitoring equivalents') for a wider range of chemicals (e.g. Hays et al, 2007; http://www.biomonitoringequivalents.net; http://www.umweltbundesamt.de/gesundheit-e/monitor/index.htm). Beyond the estimation of daily intakes provided by some existing models, the determination of internal effective 
concentrations, i.e. in the target tissues where toxic effects arise, will be required in the future to comprehensively assess 'exposure' and to characterize accurately the link between the intake from the environment and health effects. However, we are not aware of any currently available modeling tool that incorporates fate and bioaccumulation models with pharmacokinetic models in a unique modeling system. Such integration would be useful for anticipating the future frontier between exposure and hazard assessment that will result from progress in mechanistic pharmacokinetics and biomonitoring programs.

\subsection{Data for exposure modeling}

Given the large spectrum of components involved in exposure assessment, as shown in Figure 2, no systematic recommendation can be made for survey/surveillance/monitoring programs. Exposure can indeed be (partly) assessed by using information from any or all of the steps defined in the full-chain assessment system, and by merging monitoring and modeled data. However, the main difficulty that exposure modelers face for validating their models is the absence of databases gathering monitoring data on the complete chain of assessment (i.e. data for environmental media, in food products, on population behaviour and on biomonitoring). Assessing how accurately mathematical models represent the real world, i.e. the complexity of environmental or human systems, is thus difficult to conduct because empirical data are seldom consistent regarding space and time and because key input data are often lacking. This absence of integrated databases can result from historical or management reasons since environmental, socio-economic (e.g. diet composition), biomonitoring and health status follow-up are generally not managed by the same institutions. Actually, data allowing a better exploitation of exposure models sometimes exist, but not always in a format allowing them to be readily collected in a homogenous form. Another way to better exploit 
monitoring data would be to merge such data, even if sporadically available, with modelling results and/or expert judgement through e.g. Bayesian techniques. Exposure modelling results, eventually in conjunction with other existing knowledge such as gained from expert judgement, can form prior estimates of exposure. The prior distribution of exposure is updated using the measured concentrations (from historical measurements) and its associated variance to obtain the posterior probability distribution. Such approaches were commonly used in occupational exposure assessment where prior expert judgments about workers exposure are merged with exposure data, but they were rarely used in environmental exposure modeling (Tielemans et al., 2007; Ramachandran, 2008; Sottas et al., 2009).

\subsection{Uncertainty, standardisation and validation in Exposure modeling}

Integrated exposure assessment has to deal with different kinds of data (measured or predicted), different models for different compartments and more or less relevant descriptions of physico-chemical phenomena (transport, biodegradation, distribution). Uncertainty assessment is thus even more challenging in integrated exposure assessment than in integrated hazard assessment. Integrated exposure assessment relies on increasingly complex scenarios and models and involves interdisciplinary integration (e.g. as shown in Figure 2, from atmospheric long-range modeling to pharmacokinetic modeling). With this, the concept of uncertainty can be more ambiguous because it incorporates many different languages, goals and methodologies. It is therefore a major challenge to make the uncertainty concept more systematic, comprehensive, shared and understandable (Briggs, 2009). In order to overcome this challenge and to facilitate a transparent evaluation of uncertainty, a comprehensive, harmonized and structured list of criteria needs to be defined for the different components of 
uncertainty. For example, the typology of uncertainty associated with exposure assessment could be classified as scenario uncertainty, model uncertainty, parametric uncertainty and measurement uncertainty. Some attempts were undertaken to better classify the components of uncertainty (HEIMSTA, 2009) and to define a tiered approach for qualitative and quantitative uncertainty in exposure assessment (WHO, 2008). These first attempts should however be followed by the construction of operational tools for identifying the sources of uncertainty in a more transparent and systematic way for improving further visualization and communication.

In the same way as for 'uncertainty', the terms 'standardisation' and 'validation' can distinguish different aspects in exposure modeling and exposure assessment in general: (i) field validation (i.e. comparison between predicted and monitored data). Given the complexity of exposure pathways, validation is generally possible only on sub-models, but not on the complete aggregated full-chain described in Figure 2; (ii) benchmarking exercises (i.e. blind comparison of models on common scenarios); (iii) numerical validation (i.e. adequacy between user guide and software, verification of numerical schemes); (iv) documentation and transparency of tools; (v) relevance to the aim (e.g. spatial and temporal required resolution, steady-state vs dynamic scenario, analytical vs numerical solutions, mechanistic vs megression approach, deterministic vs probabilistic approach, etc).

\section{Integrated risk assessment of chemical mixtures}

The 2001 WHO report on Integrated Risk Assessment mentioned risks from exposure of humans and the environment to multiple agents via multiple routes as an important aspect. However, details of the application of the IRA concept to chemical mixtures were not worked out. In comparison to conventional single substance assessments, the assessment of the joint 
toxicity of chemicals co-occurring in an exposure scenario may already be seen as an ambitious integrative step in itself. Beyond that, however, the basic idea of IRA, i.e. the integration of human risk assessment (HRA) and environmental risk assessment (ERA) in a single process, could also be extended from single chemicals to mixtures of chemicals. With this aim, the scope for integration may encompass four specific aspects: (i) the use of a common methodology for HRA and ERA of chemical mixtures, (ii) the use of a common strategy for bridging gaps in single substance data sets that are needed for modeling human and environmental mixture toxicity, (iii) the identification of groups of substances with a common mode of action, so-called cumulative assessment groups (CAG), that are relevant in both humans and wildlife species, and (iv) the identification of common situations of joint exposure to multiple chemicals and the actual co-performance of HRA and ERA for such situations.

Two different types of approaches are used for mixture toxicity assessments: the so-called "whole mixture approach" (WMA) and the "component-based approaches" (CBA) (see Kortenkamp et al., 2009 for an overview). The WMA means that the mixture of concern is experimentally tested just like a single substance. The CBA means that the expected toxicity of a mixture is calculated on the basis of toxicity data for individual mixture components by applying appropriate models of joint action. Whole mixture testing necessarily yields results that are specific for the actually tested mixture and endpoint. Extrapolations to similar mixtures and/or endpoints may be possible only under special circumstances. CBAs make use of generic mixture toxicity concepts that can be applied to any combination of chemicals for which single substance data are available for the endpoint of concern. Therefore, CBAs lend themselves to the development of an integrated approach, both in terms of a common methodology for calculating predictions of mixture toxicity and the use of a common strategy for bridging gaps in experimental single substance toxicity data. 
Methods and models used for CBAs are essentially all based on one of only two different "non-interaction" concepts, or a combination of both: "concentration (or dose) addition" (CA) and "independent action" (IA) (also called "response addition"). CA assumes that mixture components have a similar mode of action. IA assumes that mixture components contribute to a common endpoint via dissimilar and fully independent chains of reactions. CA implies that concentrations (or doses) of substances below individual thresholds (zero effect levels) may still contribute to the overall toxicity of a mixture, while IA does not. CA and IA are well established concepts in both human and environmental mixture toxicology and have even been discussed in the context of epidemiological health risk assessments (Boedeker and Backhaus, 2010). Recently, the European Commission's Scientific Committees proposed a tiered approach for mixture risk assessment that makes use of these concepts and that is intended to be applicable for both HRA and ERA of chemical mixtures (EC, 2011).

Despite these methodological commonalities, however, there is a controversial issue where the opinions about the appropriate regulatory use of CBAs clearly differ between experts from the human and the environmental arena. This refers to the assessment of the toxicity of multicomponent mixtures of dissimilarly acting chemicals at low doses or concentrations, whereby "low" denotes levels that are considered to be regulatory acceptable for individual chemicals, such as "acceptable daily intake values" (ADI) or "derived no effect levels" (DNEL) for humans, and "predicted no effect concentrations" (PNEC) for organisms in the environment. As a recent communication from the European Commission points out, the Scientific Committees concluded that in relation to human health the level of concern about such mixture "should be assumed to be negligible", while in relation to ecological effects, such mixtures should be "considered as a possible concern" (EC, 2012).

Two of the various reasons for this dichotomy are the different types of endpoints and the different protection goals of ERA and HRA. ERA aims to protect populations of millions of 
different species and considers apical endpoints such as mortality or population growth that may be affected by a multitude of chemicals with diverse modes of action. To this end, the general assumption of CA is widely accepted as a cautious and not vastly over-protective first tier approach (ECETOC, 2011; Backhaus and Faust, 2012). HRA, in contrast, seeks to protect individual humans from adverse health outcomes that may be caused by specific types of chemicals with specific modes of action only, such as certain forms of cancer for instance. As a consequence, grouping of chemicals into appropriate CAGs is considered to be an important step for HRA of chemical mixtures, but it is often hampered by missing knowledge about the modes of action of environmentally relevant chemicals. Integrated approaches such as the AOP concept outlined in section 2.4 may play a key role for advancements in the field of grouping chemicals for human mixture risk assessments.

Toxicokinetic or toxicodynamic interactions of mixture components may result in significant deviations of the actual mixture toxicity from predictions based on the non-interaction concepts of CA and IA. Synergistic effects that are much stronger than expectable are obviously a rare event (Kortenkamp et al., 2009; Boobis et al., 2011) but they give reasons for particular regulatory concern. The search for tools that would allow identification of synergistic substance combinations in a systematic way is on-going and there is great hope that this could become achievable in the future by means of toxicogenomic approaches (Altenburger et al., 2012). Currently, however, the potential for interactions can only be evaluated on a case-by-case basis. To this end, the so-called "binary weight of evidence" approach (BINWOE) has been developed for the purpose of human health hazard assessments (Pohl et al., 2009). This is a resource intensive semi-quantitative assessment tool, which has been used by the US Agency for Toxic Substances and Disease Registry (ATSDR) for characterizing the likelihood of interactions in certain priority mixtures (www.atsdr.cdc.gov/interactionprofiles/index.asp). For the environmental hazard assessment 
of mixtures, analogous approaches are lacking at the moment. An integrated risk assessment approach may trigger corresponding advancements.

Application of physiologically based pharmacokinetic/pharmakodynamic modelling (PBPK/PD) is an advanced approach to chemical mixture toxicology (e.g. Krishnan et al., 1994; Price and Krishnan, 2011). PBPK/PD models are highly specific for a particular animal and require detailed knowledge of its physiology, such as for example the exposed skin surface or the alveolar ventilation rate. Specific data for the relevant mixture components are needed, such as blood/air, blood/tissue partition coefficients and metabolic rate constants. Krishnan et al. (1994) list some 45 parameters that build up these models. In view of these huge data demands, actual application of this approach has been confined to studies of mixtures with only a few compounds in a few selected animal test systems (Krishnan et al., 1994; Verhaar et al., 1997). In principle, the methodology is useful for both HRA and ERA of mixtures. In practice, however, applicability in the field of ERA is strongly limited by insufficient knowledge about the detailed physiology of most of the many different organisms in an ecosystem.

Similar situations of co-exposure of both humans and environmental organisms to multiple chemicals may provide starting points for performing integrated mixture hazard and risk assessments. Surface waters, for instance, may contain a complex cocktail of contaminants to which aquatic organisms are directly exposed, while exposure of humans to a similar or different mixture of the same components could occur via the consumption of drinking water or fish. As part of the development of a WHO/IPCS framework for mixture risk assessments, a case study was performed on a hypothetical mixture of 10 contaminants that have been detected in US surface water monitoring programs (Meek et al., 2011). The study was confined to the cumulative risk for humans from the use of surface water as drinking water. However, it is imaginable that such a study design could be extended from hypothetical to 
real mixtures and from an isolated HRA to an integrated assessment of the risks to both humans and aquatic organisms. Such types of in-depth case studies could help to clarify in detail, where human and ecological assessments of cumulative risks can really benefit from each other and where integration may only produce a counterproductive increase in complexity, time and costs.

\section{Integrating RA and Socio-Economic Analysis (SEA)}

\subsection{Socio-economic assessment and its status in EU Regulation}

Socio-economic assessment - or analysis - (SEA) can be regarded as a form of regulatory impact assessment used in the management of environmental and health risks. Regulatory impact analysis is used by public authorities "to balance the potential benefits and costs of action or lack of action", as mentioned in the EU treaty (Foundation for EU Democracy, 2009). The conceptual framework for environmental policy assessment is, as clearly expressed in the case of the EU by the above excerpt of the treaty, welfare economics and cost-benefit analysis. Several European directives or regulations (e.g. Water Framework Directive, Industrial Emissions Directive, REACH regulation) explicitly refer to cost-benefit analysis or SEA (ECHA, 2008).

The ambition of SEA in the context of chemicals management is to get a rational, balanced and holistic view of advantages and drawbacks of the continued use or withdrawal of chemicals that are on the market. Basically, SEA consists of an inventory and an assessment of all positive (benefits from its use) and negative impacts (originating in health and environmental risks) of the chemical, and of its alternatives, and then compares the chemical and the alternatives in terms of the overall social value (benefits minus costs) that 
they bring to society. SEA also offers the ability to handle chemical risks management in a framework that is also capable of being used to study other risks (including other environmental, health or technological risk issues), therefore improving the consistency of policymaking.

\subsection{How risk assessment can be supported by socio-economic assessment?}

The SEA framework is formulated in terms of outcomes that are understandable and matter citizens (good health, quality of life, costs). It thus helps in expressing and therefore communicating on risks and risk policy, in a way that is meaningful to non-specialized decision makers and to the public. SEA is also capable of providing integrated views of impacts across human health and the environment, thus promoting what could be called an "integration of outputs". Given that most of the EU regulation, either explicitly or in its practical implementation, still considers human health and ecosystems issues separately, better policy making can be expected when progress is made in that direction. Furthermore, SEA can work with RA on the improvement of models and methods in RA : since SEA is working on use and release of chemicals in the economy (use pattern scenarios) there could be collaboration on setting industrial or domestic use patterns and consumers or workers behaviour, by cooperation between social scientists and exposure assessors (e.g. hygienists). In the case of REACH, the two expert committees of the European Chemicals Agency respectively working on RA and SEA are actually working together to produce common opinion documents on the managements of specific chemicals for the Agency.

There are, however, many challenges to overcome before SEA can actually augment RA. First, SEA itself faces methodological and data challenges that are in some cases unlikely to be resolved in the short term (e.g. how to derive accurate costs of reducing risks in situations of asymmetric information between regulators and regulated, or how to derive the 
value of reducing risks associated with uncertain impacts on ecosystems ) SEA outputs need to be given within acceptable ranges of uncertainty and scientific consensus to deliver useful information for integrated RA and risk management. Second, a real and fruitful integration would only occur if the outcome of RA provides a relevant input for SEA. SEA requires that the assessment of effects such as reprotoxicity or neurotoxicity would be as much as possible related to endpoints that can be appraised in an economic framework, such as fertility or loss of IQ points. Several other examples of the difficulties to harmonize RA and SEA needs can be given (see (SCHER/SCENIHR/SCCS, 2011)): "risk characterisation ratios" cannot as such be assessed in socio-economic terms; RA is often looking at worst cases, in order to derive protective risk estimates for individuals or selected animal species, whereas SEA is often concerned with looking at central tendency estimates of risk and their distribution among the whole population ; SEA considers not only the substance under investigation but also substitute substances that could be used as less harmful surrogates, which means that RA for these substances would also be required. More generally, SEA promotes working on groupings that would be meaningful in a socio-economic viewpoint, in addition to physicochemical or modes of action considerations, by looking at groups that are consistent in terms of societal issues and responses.

It is thus crucial to connect the respective indicators in RA and SEA in order to share the terminologies or to build "intermediate indicators" between those used by RA (e.g. cancer risk indicators) and SEA (e.g. socio-economic impacts of cancers). Work has been initiated recently (WCA Environment, 2011; Verhoeven J.K., et al., 2012) to explore how to use concepts or tools such as LCA (life-cycle assessment) and SSD (species sensitivities distributions) to translate environmental risk assessments (ERA) results into useful inputs for SEA. 
In this context, however, producing economic estimates of the damage costs to health and the environment still raises questions that go beyond strict SEA disciplinary perspectives and practices. Insights from other disciplines are necessary in order to ensure that SEA contributes to integrated RA with more credibility and acceptance (Gezondheidsraad, 2008).

\section{3. $\quad$ Addressing issues related to social impact}

Beyond the capacity to produce economic estimates of the costs to health and environment following a risk assessment process, the challenges to contribute to a sustainable societal model involve also addressing issues related to social impact.

Indicators from RA with sociological relevance would help SEA to improve the relevance of RA. The OECD identifies in the OECD Better Life Index 11 (OECD, 2012) the following topics as essential in the areas of material living conditions and quality of life: housing, income, jobs, community, education, environment, civic engagement, health, life satisfaction, safety and work-life balance. Relevant aspects could be captured in indicators which reflect inter alia elements specific to value creation for the society as whole, like: improving living conditions; creating opportunities for certain categories of people, in terms of economic growth and jobs; developments in terms of technology and knowledge; maintenance or improvement of certain ecosystem services; health benefits; etc. When focusing on chemicals, more detailed and precise indicators could be identified, like in the case of pesticides or fertilisers, their contribution to aspects of food security, poverty, and malnutrition alleviation and eradication. Food security indicators should cover aspects related to availability (including energy and protein supply), physical access to food, and economic access and utilization (FAO, 2012). With reference to environmental assessment, indicators can be derived to feed into the evaluation of the impact on ecosystem services. The concept of 
the ecosystem services can be addressed using the four categories of services identified by the Millennium Ecosystem Assessment (Millennium Ecosystem Assessment, 2005): provisioning services, regulating services, habitat and supporting services and cultural services. Some of the impacts on ecosystem services might already be captured in the IRA through the protection goals assumed in the assessment process (i.e. air quality; quality of water or protecting biodiversity) while others remain outside the assessment process generated by the regulatory framework (i.e. provision of food and raw materials; carbon sequestration and storage; impact on climate change; soil fertility; recreation and mental and physical heath; aesthetic appreciation; cultural aspects; etc.). The evaluation of these impacts would help decision makers derive a more informed decision as well as considering the costs and benefits for society of their decision and establishing the appropriate trade-offs.

A relevant contribution to RA by sociological aspects could also be addressing the gap between risk assessment and risk perception, social scientists being able to study and reveal risk and benefits perceptions in the society and in certain cases using certain methodologies to evaluate the changes in the perceptions on specific risks (Dreyer et al., 2010). Hence, SEAs would not only rely on "scientific" risk estimates from RA but also be able to predict societal perception of risks, thereby enriching the economic concepts of value, and supporting risk managers to better communicate on risks and mitigation measures. Extensive experience and scientific literature exist in this area, showing the importance of perceptions dimensions that are not reducible to economic value formation (see for instance Gupta et al., 2012 and Satterfield et al., 2009 for a discussion in the case of nanomaterials).

When social values are to be studied, the point of view from political science perspectives could be clarified with the involvement of social scientists as well as ethicists (for instance, consideration of animal testing issues). At this stage, foresight on various changes to policy frameworks required in the future and impacted by the IRA and SEA 
process would definitely provide useful information. However, it is important to remember that, even when augmented with the recourse to those many expert categories, SEA should not be used as a substitution to political decision on risk management priorities in a society.

\section{Conclusions and future challenges}

This paper describes the scope and prospects of the integrated assessment and of including socio-economic analysis in this risk assessment. A number of recommendations for the next research steps can be derived from this analysis. Integrated hazard assessment would benefit from developments and validations of ITS able to synthesize information from test and non-test methods, with or without extrapolation between species or levels of biological organizations. To improve the use and the relevance of these ITS, data extrapolation methods are available, based on modeling (PBPK models for instance) and we should consider modes of action to support the integration of information across human and environmental endpoints. The first step will be to assess whether existing methods and WoE schemes can address this assessment of mode of action adequately, or whether additional research is needed.

Exposure assessment would require new developments to relate the exposure concentrations for the different routes of exposure, the measurements from biomonitoring and relevant internal concentrations, and by linking with hazard assessment. Again, generic PBPK models would allow the goal to be achieved.

Initiatives should also be started, that aim at a better exchange between RA and SEA in order to better inform about risk management options available following the risk assessment.

Clear opportunities for demonstrating the value of integration have been identified for mixture RA, such as the use of a consistent methodology for both human RAs and 
environmental RAs of chemical mixtures, based on generic approaches and common principles, as well as the consistent use of methodologies for extrapolating between different individual chemicals and different endpoints in order to bridge gaps in single substance data sets.

The HEROIC project has been designed to help identify and answer the needs for IRA. It will contribute to the practical implementation of IRA by building a central knowledge platform to create awareness for and facilitate access to human and environmental RA data; by promoting the use of non-test methods to extrapolate data or generate new ones; by considering the relevance of key concepts such as MOA and AOP to support the use of environmental toxicity data as a substitute for human/animal toxicity data, and vice versa; by promoting a dialogue between RA and SEA. HEROIC works in a more favourable environment compared to previous initiatives since the accessibility of new data sets generated under REACH will facilitate the cross-comparability of human and environmental risk assessment data. Since previous case studies trying to highlight the potential benefits of IRA have not had a significant impact, it is of importance that real-life case studies will be developed to fully demonstrate the potential and relevance of IRA, in particular with regard to cost-benefits analysis, and maximize its use in future risk assessment procedures at the regulatory level.

\section{Acknowledgments}

This work was supported by the European Commission through the Project HEROIC (FP7-282896) (Coordination Action funded under the 7th Framework Program). The authors would also like to thank two anonymous reviewers who helped to improve the manuscript, and all contributors to the first HEROIC expert meeting and HEROIC members for fruitful discussions on topics related to the contents of this manuscript: J. Tarazona, N. Carmichael, 
N. Roth, B. Richard, D. Barcelo, P. Grasso, T. Vermeire, D. Demortain, M. Junghans, R. Fautz, D. Kroese, R. Beaudouin, F. Bois, M. Farre, F. Brion, S. Ait Aissa, M. Bisson, T. Porsbring, A. Ragas, J.-L. Dorne, T. Frische, S. Ronga, T. Ginebreda, M. Grote, L. Posthuma, L. Geoffroy, A. James, C. Kairo, R. Smolders, L. Sparfel, C. Legind, F. Zeman, R. Glass, R. Bonnard, T. Tanaka, A. Crépet, N. Pucheux, A. Kortenkamp, L. Papadaki, K. Machera, K. Kyriakopoulou, Alan Boobis, James (Jim) Bridges, Myriam Jacobs, Georges Loizou, Mark Montforts, Peter Wiedemann. HEROIC consortium member R. Glass kindly provided editorial feedback for the manuscript.

\section{References}

Agerstrand M, Kuster A, Bachmann J, Breitholtz M, Ebert I, Rechenberg B, Ruden C. Reporting and evaluation criteria as means towards a transparent use of ecotoxicity data for environmental risk assessment of pharmaceuticals. Environ Poll 2011;159:2487-92.

Ahlers J, Stock F, Werschkun B. Integrated testing and intelligent assessment - new challenges under REACH. Environ Sci Pollut Res 2008;15:565-72.

Altenburger R, Scholz S, Schmitt-Jansen M, Busch W, Escher BI. Mixture toxicity revisited from a toxicogenomic perspective. Environ Sci Technol 2012;46:2508-22.

Ankley GT, Bennett RS, Erickson RJ, Hoff DJ, Hornung MW, Johnson RD, et al. Adverse outcome pathways: A conceptual framework to support ecotoxicology research and risk assessment. Environ Toxicol Chem 2010;29:730-41.

Backhaus T, Faust M. Predictive environmental risk assessment of chemical mixtures: a conceptual framework. Environ Sci Technol 2012;46:2564-73.

Benigni R, Bossa C. Mechanisms of Chemical Carcinogenicity and Mutagenicity: A Review with Implications for Predictive Toxicology. Chem Rev 2011;111:2507-36. 
Bhattacharya S, Zhang Q, Carmichael PJ, Boekelheide K, Andersen ME Toxicity Testing in the $21^{\text {st }}$ Century: Defining New Risk Assessment Approaches Based on Perturbation of Intracellular Toxicity Pathways. PLoS One 2011;6(6):e20887, doi:10.1371/journal.pone.0020887.

Blaschke U, Eismann K, Böhme A, Paschke A, Schüürmann G. Structural Alerts for the Excess Toxicity of Acrylates, Methacrylates and Propiolates derived from Their Short-Term and Long-Term Bacterial Toxicity. Chem Res Toxicol 2012;25:170-80.

Boedeker W, Backhaus T. The scientific assessment of combined effects of risk factors: different approaches in experimental biosciences and epidemiology. Eur $\mathrm{J}$ Epidemiol 2010;25:539-46.

Böhme A, Thaens D, Schramm F, Paschke A, Schüürmann G. Thiol Reactivity and its Impact on the Ciliate Toxicity of $\alpha, \beta$-Unsaturated Aldehydes, Ketones and Esters. Chem Res Toxicol 2010;23:1905-12.

Boobis A, Budinsky R, Collie S, Crofton K, Embry M, Felter S, et al. Critical analysis of literature on low-dose synergy for use in screening chemical mixtures for risk assessment. Crit Rev Toxicol 2011;41:369-83.

Bradbury S, Feijtel T, Van Leeuwen K. Meeting the scientific needs of ecological risk assessment in a regulatory context. Environ Sci Technol 2004;38:463a-70a.

Briggs D, Sabel C, Lee K. Uncertainty in epidemiology and health risk and impact assessment. Environ Geochem Health 2009;31:189-203.

Briggs K, Cases M, Heard DJ, Pastor M, Pognan F, Sanz F, et al. Inroads to predict in vivo toxicology-An introduction to the eTOX project. Int J Mol Sci 2012;13:3820-46.

Buist H, Aldenberg T, Batke M, Escher S, Klein Entink R, Kühne R, et al. The OSIRIS Weight of Evidence approach: ITS mutagenicity and ITS carcinogenicity. Regul Toxicol Pharmacol 2013; in press, online available, DOI 10.1016/j.yrtph.2013.01.002. 
Combes R, Balls M. Intelligent testing strategies for chemicals testing - a case of more haste, less speed? ATLA 2005;33:289-97.

Davis JA, Gift JS, Zhao QJ. Introduction to benchmark dose methods and U.S. EPA's benchmark dose software (BMDS) version 2.1.1. Toxicol Appl Pharmacol 2011;254:181-91.

Dimitrov S, Dimitrova G, Pavlov T, Dimitrova N, Patlewicz G, Niemela J, et al. A stepwise approach for defining the applicability domain of SAR and QSAR models. J Chem Inf Model 2005;45:839-49.

Dreyer M, Renn O, Cope S, Frewer LJ. Including social impact assessment in food safety governance, Food Control 2010;21:1620-8.

EC. Toxicity and Assessment of Chemical Mixtures (Final approved opinion); 2011.

EC. The combination effects of chemicals - Chemical mixtures. Communication from the Commission to the Council, COM(2012) 252 final, Brussels, 31.5.2012; 2012.

ECETOC. Toxicological Modes of Action: Relevanve for Human Risk Assessment. Technical Report No. 99, Brussels, Belgium, 67 pp; 2006.

ECETOC. Development of guidance for assessing the impact of mixtures of chemicals in the aquatic environment. Technical Report No. 111, www.ecetoc.org, Brussels, Belgium; 2011.

ECETOC. Category approaches, Read-across, (Q)SAR. Technical Report No. 116, Brussels, Belgium, 183 pp; 2012.

ECHA. Guidance on Socio-Economic Analysis - Restrictions. ECHA Report; 2008.

ECHA. Guidance on information requirements and chemical safety assessment. Chapter R.7c: Endpoint specific guidance. ECHA-12-G-23-EN; 2012.

Foundation for EU Democracy, "The Lisbon Treaty : The readable version”, Third Edition; 2009. 
FAO, WFP and IFAD. The State of Food Insecurity in the World 2012. Economic growth is necessary but not sufficient to accelerate reduction of hunger and malnutrition. Rome, FAO; 2012.

Gezondheidsraad (Health Council of the Netherlands). Prudent Precaution; 2008.

Gupta N, Fisher ARH, van der Lans IA, Frewer LJ. Factors influencing societal response of nanotechnology: an expert stakeholder analysis. J Nanopart Res 2012;14:857.

Hays SM, Becker RA, Leung HW, Aylward LL, Pyatt DW. Biomonitoring equivalents: A screening approach for interpreting biomonitoring results from a public health risk perspective. Regul Toxicol Pharmacol 2007;47:96-109.

HEIMSTA. Conceptual framework and scoping report for uncertainty analysis and integration of uncertainty methods into four case studies. HEIMSTA project; Deliverable 1.2.1.; 2009.

Janer G, Hakkert BC, Piersma AH, Vermeire T, Slob W. A retrospective analysis of the added value of the rat two-generation reproductive study versus the rat subchronic toxicity study. Reprod Toxicol 2007;24:103-13.

Jaworska J, Gabbert S, Aldenberg T. Towards optimization of chemical testing under REACH: A Bayesian network approach to Integrated Testing Strategies. Regul Toxicol Pharmacol 2010;57:157-67.

Ji L, Schüürmann G. Computational Evidence for $\alpha$-Nitrosamino Radical as Initial Metabolite for Both the P450 Dealkylation and Denitrosation of Carcinogenic Nitrosamines. J Phys Chem B 2012a;116:903-12.

Ji L, Schüürmann G. Model and Mechanism: N-hydroxylation of Primary Aromatic Amines by Cytochrome P450. Angew Chem Int Ed 2013;52:744-48.

Klimisch HJ, Andreae M, Tillmann U. A systematic approach for evaluating the quality of experimental toxicological and ecotoxicological data. Regul Toxicol Pharmacol 1997;25:1-5. 
Kortenkamp A, Backhaus T, Faust M. State of the art report on mixture toxicity. Contract study prepared for the European Commission, DG Environment, study contract no. 070307/2007/485103/ETU/D.1; available online from the website of the European Commission at http://ec.europa.eu/environment/chemicals/pdf/report_Mixture toxicity.pdf; 2009.

Krishnan K, Andersen ME, Clewell HJ, Yang RSH. Physiologically based pharmacokinetic modeling of chemical mixtures. In: RSH Yang (ed.) Toxicology of chemical mixtures: case studies, mechanisms and novel approaches, pp. 399-437. Academic Press, San Diego, CA; 1994.

Kühne R, Ebert R-U, Schüürmann G. Chemical Domain of QSAR Models from Atomcentered Fragments. J Chem Inf Model 2009;49:2660-9.

Lalko JF, Kimber I, Dearman RJ, Gerberick F, Sarlo K, Api AM. Chemical reactivity measurements: Potential for characterization of respiratory chemical allergens. Toxicol In Vitro 2011;25:433-45.

Meek ME, Boobis AR, Crofton KM, Heinemeyer G, Van Raaij M, Vickers C. Risk assessment of combined exposure to multiple chemicals: A WHO/IPCS framework. Regul Toxicol Pharmacol 2011;60:S1-S14.

Mekenyan O, Patlewicz G, Dimitrova G, Kuseva C, Todorov M, Stoeva S, et al. Use of Genotoxicity Information in the Development of Integrated Testing Strategies (ITS) for Skin Sensitization. Chem Res Toxicol 2010;23:1519-40.

Millennium Ecosystem Assessment, Ecosystems and Human Well-being: Synthesis. Island Press, Washington, DC; 2005.

Mulliner D, Wondrousch D, Schüürmann G. Predicting Michael-Acceptor Reactivity and Toxicity through Quantum Chemical Transition-State Calculations. Org Biomol Chem 2011;9:8400-12. 
Nestorov I. Whole body pharmacokinetic models. Clin Pharmacokinet 2003;42:883-908.

OECD. Guidance on Grouping of Chemicals. OECD Environment Health and Safety Publications, Series on Testing and Assessment No. 80, 99 pp; 2007.

OECD. Guidance Document for using the OECD (Q)SAR Application Toolbox to develop Chemical Categories according to the OECD Guidance on Grouping of Chemicals, OECD Environment Health and Safety Publications, Series on Testing and Assessment No. 80, 118 pp; 2009.

OECD. Report of the Workshop on Using Mechanistic Information in Forming Chemical Categories, 8-10 December 2010, Crystal City VA, USA. Series on Testing and Assessment No. 138. 18 May 2011; 2011.

OECD. Appendix I. Collection of working definitions. http://www.oecd.org/chemicalsafety/testingofchemicals/49963576.pdf; 2012.

OECD. Better Life Index, retrieved on $20^{\text {th }}$ of December 2012 from http://www.oecdbetterlifeindex.org/; 2012.

OSIRIS. Optimized Strategies for Risk Assessment of Industrial Chemicals through Integration of Non-Test and Test Information. EU Project, contract no. GOCE-CT-2007037017, 2007-2011. OSIRIS project website: http://www.osiris-reach.eu/. OSIRIS webtool website: http://osiris.simpple.com/OSIRIS-ITS/welcome.do $; 2011$.

Péry ARR, Henegar A, Mombelli E. Maximum-Likelihood Estimation of Predictive Uncertainty in Probabilistic QSAR Modeling. QSAR Comb Sci 2009;28,338-44.

Péry ARR, Desmots S, Mombelli E. Substance tailored testing strategies in toxicology: An in silico methodology based on QSAR modeling of toxicological thresholds and Monte Carlo simulations of toxicological testing. Regul Toxicol Pharmacol 2010;56:82-92.

Pohl HR, Mumtaz MM, Scinicariello F, Hansen H. Binary weight-of-evidence evaluations of chemical interactions - 15 years of experience. Regul Toxicol Pharmacol 2009;54:264-71. 
Price K, Krishnan K. An integrated QSAR-PBPK modelling approach for predicting the inhalation toxicokinetics of mixtures of volatile organic chemicals in the rat. SAR QSAR Environ Res 2011;22:107-28.

Przybylak KR, Madden JC, Cronin MTD, Hewitt M. Assessing toxicological data quality: basic principles, existing schemes and current limitations. SAR QSAR Environ Res 2012;23:435-59.

Punt A, Schiffelers M-JWA, Horbach GJ, van de Sandt JM, Groothuis GMM, Rietjens IMCM, et al. Evaluation of research activities and research needs to increase the impact and applicability of alternative testing strategies in risk assessment practice. Regul Toxicol Pharmacol 2011;61:105-14.

Ramachandran G. Toward Better Exposure Assessment Strategies-The New NIOSH Initiative. Ann Occup Hyg 2008;52:297-301.

Satterfield T. and al., "Anticipating the perceived risk of nanotechnologies", Nature Nanotech 2009;4:752-58.

SCHER/SCENIHR/SCCS. Improvement if Risk Assessment in View of the Needs of Risk Managers and Policy Makers; 2011.

Schneider K, Schwarz M, Burkholder I, Kopp-Schneider A, Edler L, Klinsner-Ovaskainen A, Hartung T, Hoffman S. "ToxRTool", a new tool to assess the reliability of toxicological data. Toxicol Lett 2009;189:138-44.

Schramm F, Müller A, Hammer H, Paschke A, Schüürmann G. Epoxide and Thiirane Toxicity In vitro with the Ciliates Tetrahymena pyriformis - Structural Alerts Indicating Excess Toxicity. Environ Sci Technol 2011; 45: 5812-9.

Schultz TW, Hewitt M, Netzeva TI, Cronin MTD. Assessing applicability domains of toxicological QSARs: Definition, confidence in predicted values, and the role of mechanisms of action. QSAR Comb Sci 2007; 26: 238-54. 
Schüürmann G, Ebert R-U, Chen J, Wang B, Kühne R. External Validation and Prediction Employing the Predictive Squared Correlation Coefficient - Test Set Activity Mean vs Training Set Activity Mean. J Chem Inf Model 2008; 48: 2140-5.

Schüürmann G, Ebert R-U, Kühne R. Quantitative read-across for predicting the acute fish toxicity of organic compounds. Environ Sci Technol 2011;45:4616-22.

Schüürmann G, Ebert R-U, Nendza M, Dearden JC, Paschke A, Kühne R. Predicting faterelated physicochemical properties. In: Van Leeuwen K, Vermeire T (eds): Risk assessment of chemicals: An Introduction. $2^{\text {nd }}$ Edition. Springer, Dordrecht, The Netherlands, Chapter 9, pp. 375-426; 2007.

Schwöbel JAH, Koleva YK, Enoch SJ, Bajot F, Hewitt M, Madden JC, et al. Measurement and Estimation of Electrophilic Reactivity for Predictive Toxicology. Chem Rev 2011;111:2562-96.

Sottas P-E, Lavoué J, Bruzzi R, Vernez D, Charrière N, Droz P-O. An empirical hierarchical Bayesian unification of occupational exposure assessment methods. Stati Med 2009; 28:7593

Spielmann H, Sauer UG, Mekenyan OG. A Critical Evaluation of the 2011 ECHA Reports on Compliance with the REACH and CLP Regulations and on the Use of Alternatives to Testing on Animals for Compliance with the REACH Regulation. ATLA 2011; 481-93.

Tebby C, Mombelli E. A kernel-based method for assessing uncertainty on individual QSAR predictions. Mol Inf; 2012;31:741-51.

Thaens D, Heinzelmann D, Böhme A, Paschke A, Schüürmann G. Chemoassay Screening of DNA-reactive Mutagenicity with 4-(4-Nitrobenzyl)pyridine - Application to Epoxides, Oxetanes and Sulfur Heterocycles. Chem Res Toxicol 2012;25:2092-2102.

Thalheim T, Vollmer A, Ebert R-U, Kühne R, Schüürmann G. Tautomer Identification and Tautomer Structure Generation Based on InCHI Code. J Chem Inf Model 2010;50:1223-32. 
Tielemans E, Warren N, Schneider T, Tischer M, Ritchie P, Goede H, et al. Tools for regulatory assessment of occupational exposure: development and challenges. J Expo Sci Environ Epidemiol 2007; 17:S72-S80.

Tluczkiewicz I, Batke M, Kroese D, Buist H, Aldenberg T, Pauné E, et al. The OSIRIS Weight of Evidence approach: ITS for the endpoints repeated-dose toxicity (RepDose ITS). Regul Toxicol Pharmacol 2013; in press, online available, DOI 10.1016/j.yrtph.2013.02.004. Verhaar HJM, Morroni JR, Reardon KF, Hays SM, Gaver Jr DP, Carpenter RL, et al. A proposed approach to study the toxicology of complex mixtures of petroleum products: The integrated used of QSAR, lumping analysis and PBPK/PD modelling. Environ Health Perspect 1997;105:179-95.

Verhoeven JK. From risk assessment to environmental impact assessment of chemical substances, Methodology development to be used in socio-economic analysis for REACH. RIVM Report 601353002/2012; 2012.

Von der Ohe PC, Kühne R, Ebert R-U, Altenburger R, Liess M, Schüürmann G. Structural alerts - A new classification model to discriminate excess toxicity from narcotic effect levels of organic compounds in the acute daphnid assay. Chem Res Toxicol 2005;18:536-55.

WCA Environment. Refinements of environmental risk assessment outputs for use in socioeconomic impact assessment under REACH. Report for the Luxembourg Environment Agency; 2011

Weed LW. Weight of Evidence: a review of concept and methods. Risk Anal 2005;25:154557.

WHO. Integrated Risk Assessment. Report Prepared for the WHO/UNEP/ILO International Programme on Chemical Safety; 2001. 
WHO. Uncertainty and data quality in exposure assessment. http://www.who.int/ipcs/publications/methods/harmonization/exposure_assessment.pdf; 2008.

Wondrousch D, Böhme A, Thaens D, Ost N, Schüürmann G. Local Electrophilicity Predicts the Toxicity-Relevant Reactivity of Michael Acceptors. J Phys Chem Lett 2010;1:1605-10. 
Macromolecules

Cell

Organ

Organism

Population

$\begin{aligned} & \text { Molecular } \\ & \text { initiating event } \\ & (\text { receptors, DNA,...) }\end{aligned} \mid \rightarrow$

Perturbations
at cell level
(proteins,
transcription)

$\rightarrow \begin{aligned} & \text { Altered } \\ & \text { function or } \\ & \text { development }\end{aligned}$

\begin{tabular}{l} 
Reproduction \\
Cancer \\
Death \\
\hline
\end{tabular}

Abundance

Structure

Figure 1. Schematic representation of a sequence of steps representing an adverse outcome pathway (AOP) 


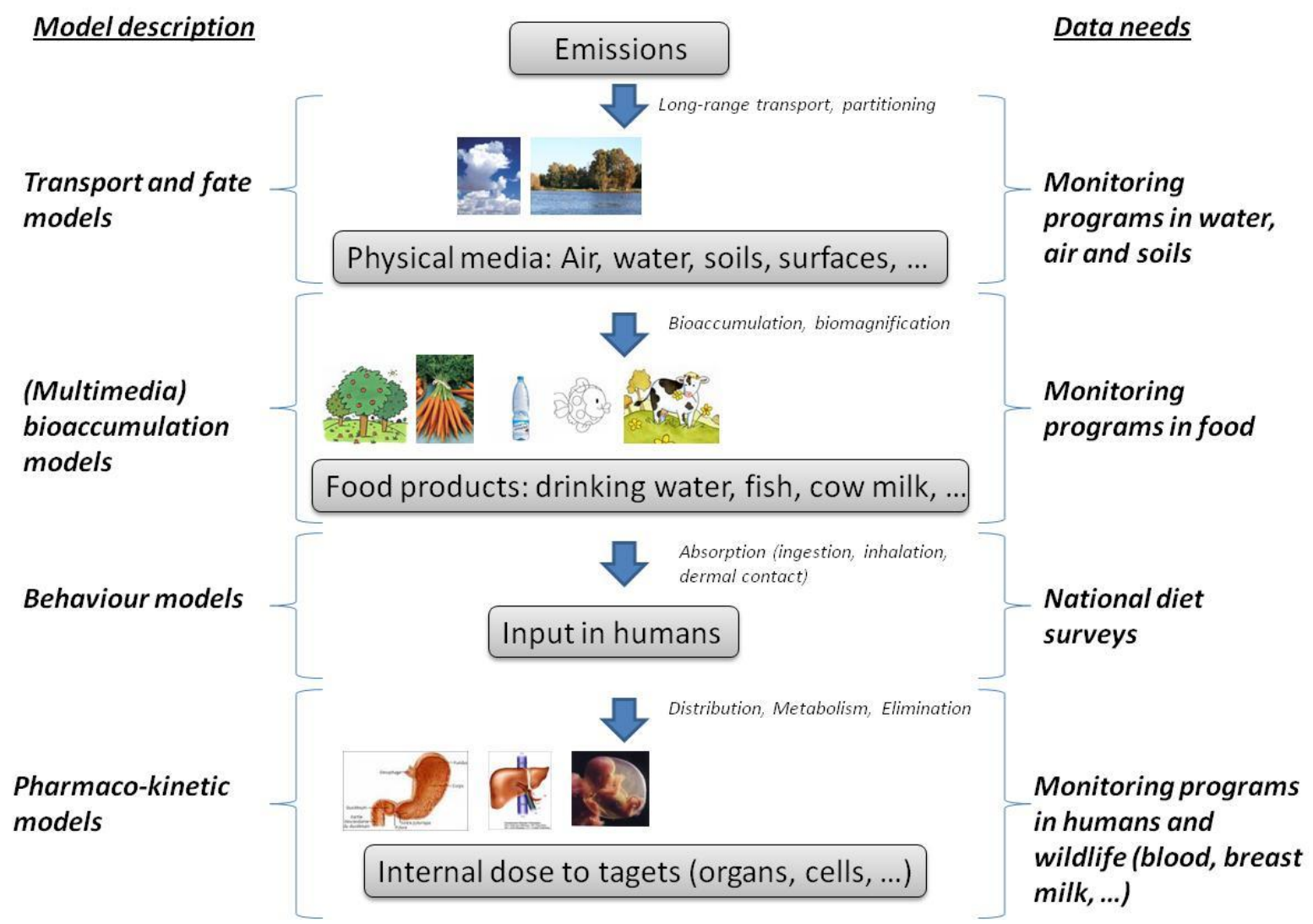

Figure 2 - Successive stages involved in Exposure assessment 